\title{
MEMBRANES AS ACCEPT JRS FOR PALMITOYL COA IN FATTY ACID BIOSYNTHESIS
}

\author{
M. SUMPER and H. TRALULE \\ Max-Planck-Institut fïr biophysikalische Chemic, \\ 3400 Götingen-Nikolausberg. Germany' \\ Reecived \&. Deceniber 1972 \\ Revised version received 20 December 1972
}

\section{Introduction}

The biosynthesis of phospholipids involves an inferesting interplay between ble and membranebound enzymes. The final assembly of phospholipid molecules is cutalyzed by nembrane-bound enzymes [1] : however the precursor substrates, palmitoyl CoA and stearoyl CoA , are synthesized (in ycast) by the soltible multienzy me complex tatty acld synthetase (FAS). As is well known palmitoyl CoA assuciates easily and unspeciffeally with a varie ty of soluble en$2 y$ mes and causes their denaturation $[2,3]$. In lins in th this, the present study shows that newly synthe. sied paimitoyl CoA cannot freely dissutiate from the FAS. Therefore a iree diffusion of individual palmity CoA molecules is highly improbable.

Thus the question arises how is paltmitoyl CoA tiansferred trom the FAS to the subsequent menbranc-bound enzy mes. The rate and mechanism of this transfer may important for the regulation of the lipid biosy nthesis. The simplest mechanism might be the transfer through direct collisions between the FAS and the membrane-hound enzymes. A slightly more sophisticated one might involve lipid binding molecules, like bevine serum albumin (BSA), uperating as carriors of paltritoyl COA. In botli cases the thansfer would be aceoniplished through the thresdimensional diffusion of macromolecules.

\footnotetext{
"The term "palmitoyl con" will be used below to denole bisth compounds.
}

Alternatively, it is conceivable tiat a combined mechanism opcrates in which palnitoyl CoA is intro" duced into target membranes and diffuses in the plane of the nembrane (cf. Adam and Delbruck [4]).This model presupposes that i) lipid membranes can serve as aceeptors (and as a reservoir) for palmitoyl CoA and ii) that the palmitoyl COA molecules can rapidly ditfuse within the plane of the membrane. The present paper provides experinental evidence in favour of this mechanism.

\section{Materials and methods}

The assiay and purification of the tatty acid synthe. tase frum yeast followed procectures described in [5]. Aceryl COA, matomyl CoA and palmitoyl COA were prepared according to $[6$ 4 1 .

BSA, CaASH and NADPH were commertial products from Bohringwerke, Marburg and Boetringer, Mannleim, respectively. $\left[{ }^{14} \mathrm{C}\right]$ Acetic anlydricle was obtained from the Radiochemical Centre. Anersham, and 1-anilino-napththalene-8-sulfonate (ANS *) from Pierce Chemicals.

E. coli K 1062 plasma trembranes were kindly supplied by Dr. P. Overath, Kisin. They were prepared according to Kaback's me thod [10].

Dimyristoyllecithin was a product from Koch Lught. The lipids were ultrasonically dispersed at $30^{\circ}$ for $3 \% 5$ min to yicld optically clear solutions.

The kine tics of palmitoyl CoA synthesis was mea. sured by following NADPl: comsumption using a 


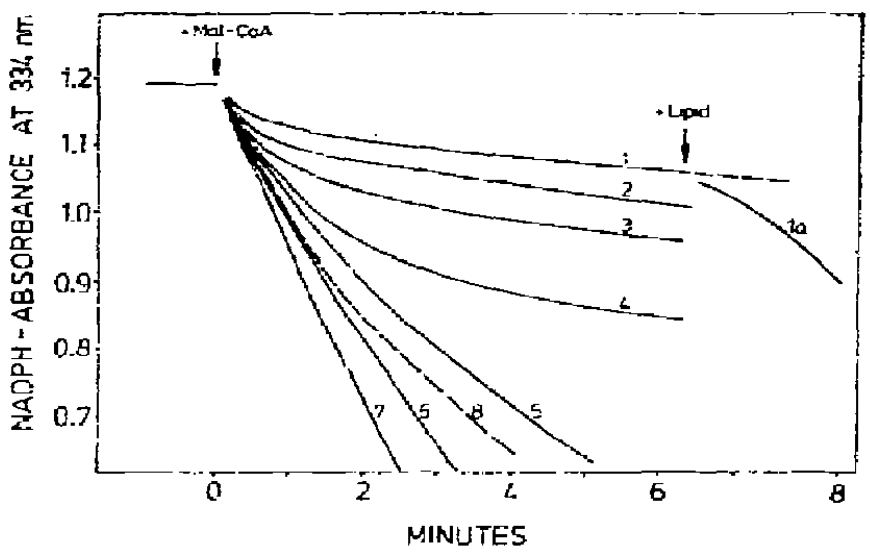

Fig. 1. Effect of lipid acceptor sub;tances on the kinetics of fatly acid syithesis (as measured by the. NADPH consumption). The incubation mix ture ( 2 ml) contained: $200 \mu$ moles

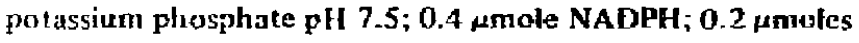
acetyl CoA and $20 \mu \mathrm{g}$ fatty avid synthetase (2009 $\mathrm{mU} / \mathrm{mg})$. The reaction was initiated by the addition of $0.2 \mu$ mote malony CoA. Curve (1): Incubation mix ture without acceptor substances; (1a): After a prolonged period of salf-inhibiion dimy nistoyllecithin bilayers were added $\left(3.8 \times 10^{-5} \mathrm{M}\right.$ lipid). Curves (2)-(6): increasing concentrations of dinyzistoyllecithin dispersions: (2) $3.1 \times 10^{-6} \mathrm{M} ;(3) 6.2 \times 10^{-6} \mathrm{M}$; (4) $12.5 \times \div 0^{-5} \mathrm{M}$ (5) $5 \times 10^{-5} \mathrm{MF}$; (6) $1.1 \times 10^{-4}$ M. Curve (7): In the iresence of $1 \mathrm{mg}$ bovine serum albumin (about $\left.7.5 \times 10^{-63} \mathrm{M}\right)$. Curve (8): In the presence of $E$. coli plasm: membranes in a concentration corresponding to $2.5 \times 10^{-5} \mathrm{M}$ lipid.

Cary 14. A total of $14 \mathrm{NADPH}$ molecules is consumed for I palmitoyl CoA. The fluorescence data were obtained with a Fica 55, an instrument allowing the recording of fully corrected differential spectra.

\section{Results}

Palmitoyl CoA and stearoyl $\mathrm{C}$ oA, the products of the FAS, are tffective inhibitcrs of the enzy matic activity of FAS [II]. This is demonstrated by curves 1 and 7 in fig. 1 . These curves show the kinetics of the fatty acid synthesis in the presence (curve 7) and in the absence (curve 1) of BSA which is a powerful anceptor of fatty acid derivatives. In the absence of BSA the enzymatic activity is blocked almost completely after a short period of synthess (rest activity about $5 \%$ ); this initial period of synthesis will be denoted in the following as the "active period". In the

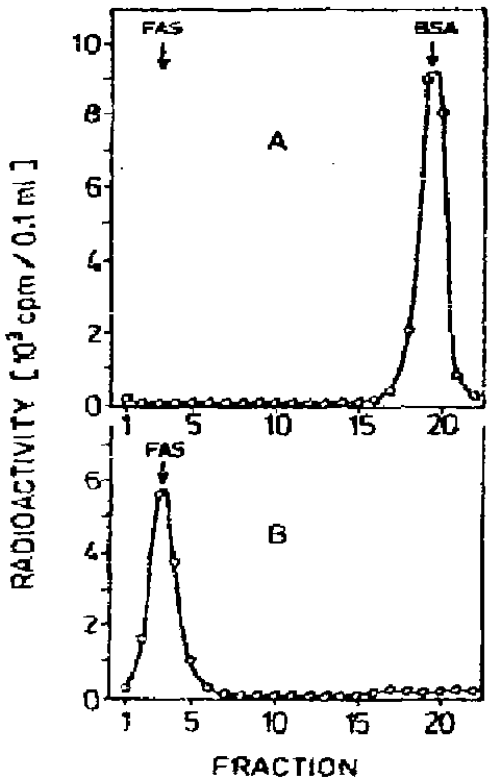

Fij. 2. Assay for the associalion of the products of fatty acid synthesis (long-chain $1^{14} \mathrm{Clacy}(\mathrm{CoN}$ ) with the fatty acid synthatase (FAS) and bovine serim albumin (BSA) by sucrose, density gradient centrifugation. The incubation mix ture ( 2 al) containecl: $200 \mu$ moles potassiun phosphate pH 7.5: 0.41 mote NADPH; $0.018 \mu$ mole $\int^{14} \mathrm{C}$ facetyl CoA $(10$ $\mu 0 . \mathrm{i} / \mathrm{mmole}$ ) and $100 \mathrm{\mu g}$ fatty acid syr the tase $(2000 \mathrm{mU} / \mathrm{mf})$ The reaction wasi initiated by the addition of $0.2 \mu$ mole mislonyl CoA. Incubation: 5 min at $25^{\circ}$. (A) Incubation in lus presence of $2 \mathrm{mg}$ BSA. (B) Without BSA (product-inhilition occlirs soon). Aliquots of the incubation mixtures wers Iayered on a linear sucrose density gradient $(5-20 \%)$ in 0. 1. M potassium phosphate pH 6.5. After centrifugation fo: $5.5 \mathrm{hr}$ at $40000 \mathrm{rpm}$ and $10^{\circ}$ (SW 40 , Beckman $e^{2-65}$ ) the fractions were analysed for long chain $\left({ }^{14} \mathrm{Clacyl} \mathrm{CoA}\right.$ in itre following way: $0.2 \mathrm{mg}$ BSA and $2 \mathrm{mi} 5 \%$ trichioroacetic atid were added to each fraction; the precipitates we re collected on millipore filters (HAWR $0.45 \mathrm{\mu m}$ ) washed 6 times with $5 \%$ trichloroacetic acid and assayed for radioactivity after drying.

presence of BSA the effect of product-inhibition is not observed. It can be shown that the paimitoyl CoA molecules are readily transferred from the FAS to BSA and that they remain quantitatively attacled to the FAS in the absence of BSA. Using $\left[{ }^{14} \mathrm{C}\right]$ acetyl $\mathrm{CoA}$ as substrate the synthesis was car ried out (A) in the presence and (B) in the absence of BSA and the association and spatial distribution o: [14 CIpalmitoyl CoA was analyzed by sucrose density gradient centrifugation. The results of this analy sis are plotted in fig. 2 . In case (A) the ${ }^{14} \mathrm{Clpalmitog!}$ 


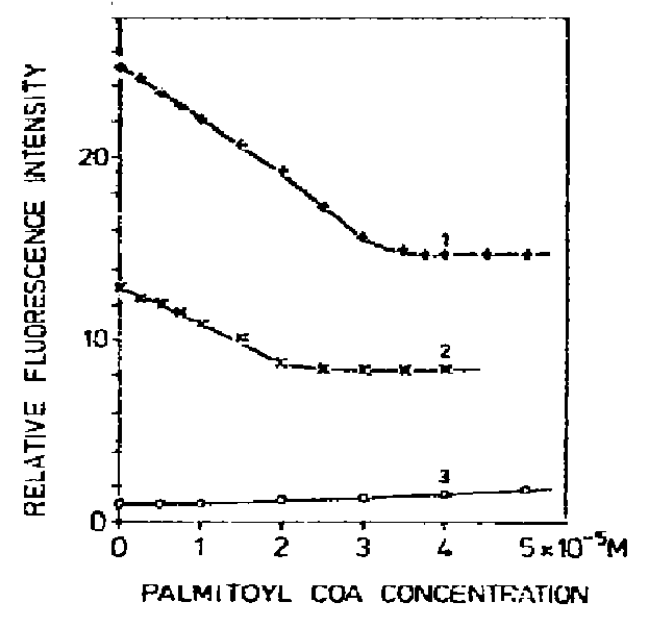

Fig. 3. Decrease in ANS $^{-}$fluorescence in tensity $(340 \mathrm{~nm}$, $500 \mathrm{~nm}$ ) upon addition of palmatoy! CoA to dimyristoyllecithin dispersions; $25^{\circ}$. ANS ${ }^{-}$conc.: $1.25 \times 10^{-4} \mathrm{M} ; 0.1 \mathrm{M}$ potassium phosphate pH 7.5. Curve (1): $1.25 \times 10^{-4} \mathrm{M}$ lexithin; Curve (2): $0.62 \times 10^{-4} \mathrm{M}$ lecithin: Curve (3): Interaction of ANS $^{-}$with palmituyl COA.

CoA sediments quantitatively with BSA whereas in case $(B)$ it remains associated with the FAS. Palmitoyl CoA was not found in detectable amounts in free solution. This suggests that dixect collisions between the FAS and BSA are necessary to transter the palmitoyl CoA molecules from the FAS to BSA. From curve $I$ of fig. I one estimates that about 125 150 palmitoyl CoA molecules are syathesized per FAS complex $\left(M . W .=2.3 \times 10^{6}\right)$ in the "active period". Thus the net production of palmitoyl CoA in a given incubation mixture during the "wetive period" is expected to intrease linearly with the enzyme concentration. This is fully supported by the relevant experiments (not shown).

Curves 2 to 6 in fig. I demonstrate that natural membranes and lipid bilayers are also powerful acceptors of long-chain acyl CoA compounds from the FAS. These curves show the kinetics of the fatty acid synthesis in the presence of increasing amounts of dimy: ijtoyllecithin bilayers. Curve 8 shows the result of anrlogous experiments in which $E$. coli plasma menbinines served as acceptors. The net anount of palniloyi COA that can be synthesized in the "active periof "increases linearly with the lipid concentration, indicating that a given lipid matrix has a well-defined capucity for the adsorption of palmitoyl CoA. From

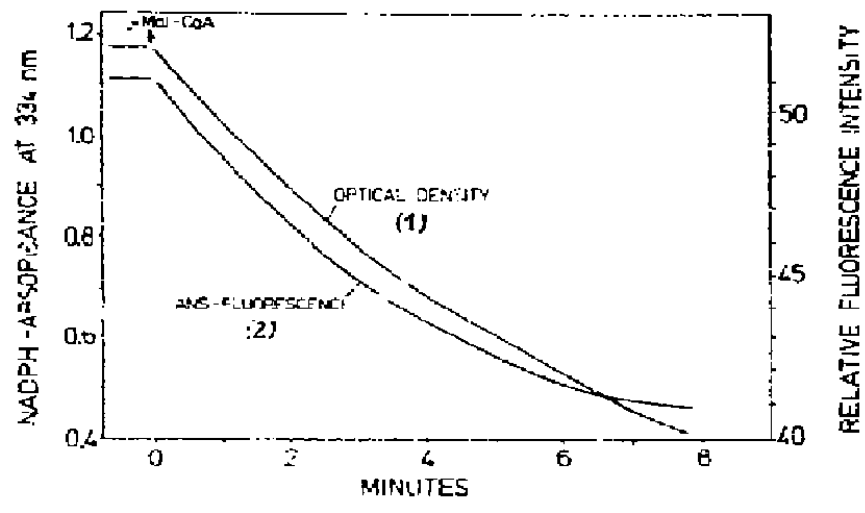

Fig- 4. Comparison w the kincics of palmitoyl CoA synthesis (1) and of the incorporation oi pilmitoyl Cof into dimy ristoylfecithin bilayers (2). Curve (2) show's the decrease in ANS $^{-}$nuorescence intensity $(410 \mathrm{~nm}, 520 \mathrm{~mm})$ in the course of the reaction (ct. fig. 3). Identical incubation mixtures wert used for both experiments containing $0.62 \times 10^{-4}$ is lipid and $5 \times 10^{-5} \mathrm{M} \mathrm{ANS}^{-}$in addition to the substrates used for experiment 1 in fig- 1 . The fluorescence decrease was mcasured against an identical incubation mix ture containing no ANS $^{-}$(differential spectrum).

thg. 1 one estimates that as much as 17-20 palmitoyl CoA molecules can be incorporated per 100 lecithin molecules.

Fig. I shows that the activity of the preduct-inhibited enzyme can be restored by the addition of phospholipid vesizles (curve la in fig. 1).

Two experiments were performed to check whether palnitoyl COA is actually incorporitied in to the lipid layers. A substitution titration was carried out using the fluorescence probe $\mathrm{ANS}^{-}$as a substituent and indicator. Second, the effect of palmitoyl CoA on the thernal phase transition of dimy ristoyllecithin was studied using optical neasurements $[13,14]$.

i) Adsorption of $\mathrm{ANS}^{-}$to lipid nembranes results in a strong enhancement of the fluorescence intensity (cf. revicw [12]). When palmitoyl CoA is adried to a lipid dispersion containing ANS" the thorescence intensity decreases, indicating the release of ANS $^{-}$fron the merrabrane surface. One obvious reason for this effect is the electrostatic repulsion of the ANS $^{-}$by the incorporated patmitoyl CoA which carries $3-4$ negative charges per molecule. As showi in fig. 3 the thusrescence intensity reaches a plateau for palmitoyl CoA concentrations larger than a value $c^{*}$, characteristic for the lipid concentration. From the values of 
$c$ determined by the curves in fig. 3 we estimate that a miximum nunber of 25 palmitoyl CoA molecules can be incorporated into the lipid layers per 100 lipid molecules. The fluorescence decrease accompanying the incorporation of palmitoyl CoA can be utilized to measure independently the rates of synthesis (NADPH-consumption) and incorporation of palmitoyl CoA. As demanstrated in fig- 4 these two processes are synchronized.

ii) Addition of palmitoyl CoA to dispersions of dimy ristoyllecithin cillses a significant broadening and a graduil diseppearance of the lipid phase transition $2: 24^{\circ}$. This effect is characteristic of the incorporati in of foreign molecules into a pure lipid matrix [15i.

\section{Discussiou}

The products of the fatty acid synthetase, palmitoyl $C o A$ and stearoyl $C o A$, remain attashed to the multienzyme complex and inhibit the enzymatic activity unless lipid accepting molecules, like BSA, or lipid membranes are present in the solution. Bila/ers of dimyristoylecithin can incorporate as much as $\mathbf{2 0}$ palmítoyl CoA molecules per 100 lipid molecules. The acceptor capacity of a given membrane is most probably determined by fixed membrane charges and by the negative charges of the incorporated palmitoyl CoA.

On the basis of our findings we propose the following mechanism for the transfer of palmitoyl CoA from the FAS to subsequent membrane-bound enzymes. In a first step the palmitoyl CoA is carried to a place somewhere on the membrane by its synthesizing en- . zyme or, perhaps, by another lipid-carrier molecule. (At present there is no evidence that the FAS is associated with membrane surfaces (18]). Lateral diffusion within the plane of the membrane would be the mechanism by which the palmitoyl COA is transferred to the subsequent membrane-bound enzymes. The following findings lend support to this hypothesis: i) In a receni study Adam and Delbruck [4] have shown that a transfer mechanism, involving lateral diffusion in addition to free diffusion permits a much faster transfer of a molecule from the cell cytoplasma to a small target on the cell membrane than.free diffusion alone. A detailed discussion of this point is given in

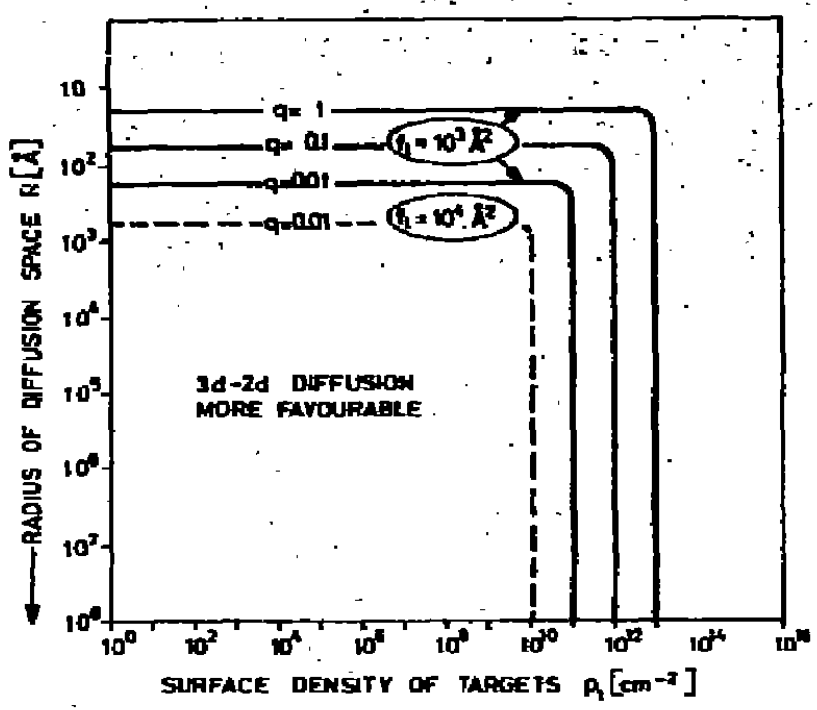

Fig. 5. Contoir lines enclosing the regime in which the combined 3d-2d fiffusion mechanism is more favourabte than free diffusion alone (calculated aceording to eq. (4), Appendix L $q$ denotes the ratio ${ }^{\prime}(3 / 2)^{/ T}(2)$ of the rupective transfer times; $f_{t}$ ts the target arca. Similair contour lines are obtained if $R$ is considered as constant and increasing values of $D_{(3)} D_{(2)}$ are drawn on the ordinate scale.

the Appendix. (ii) The occurence of rapid lateral diffusion of lipid soluble molecules in lipid bilayers and natural meribranes has been established in recent ESR studies $[16,17]$. The coefficients of lateral diffusion for androstane and fatiy acids are as high as (1-3) $\times 10^{-8} \mathrm{~cm}^{2} / \mathrm{sec}$, corresponding to a nèt displacement of these molecules by about $10000 \AA$ in one second. Therefore palmitoyl CoA molecules are also expected io be highly mobile within the plane of a lipid matrix. In addition to a higher transfer rate. the combined mechanism would also avoid complications due to the unspecific association of palmitoyl CoA with soluble proteins within the cell (detergent properties of palmitoyl CoÄ).

According to this model a natural membrane containing palmitoyl CoA can be visuatized as a resevoir of substrates for the enzymes involved in the further assembly of lipid molecules (glycerolphosphate acyltransferases, desaturases, etc.). The rate of fatty acid biosynthexis would then be goveined by the acceptor capacity and the degree of saturation of the mem: brane reservoir - in close analogy to the investigated model systems. This inteplay may bè important for the regulation of the lipid biosynthesis. 
Acknowledgements

We thank Prof. F. Lynen for helpful discussions and support. The interest of Prof. M. Eigen in this work is gratefully acknowledged. We are indebted to Dr. G. Adam for expert criticism and to Dr. P. Overath for providing $E$ - coli membranes.

Apprendix: The advantage of lateral diffusion in the iransfer of palmitoyl CoA from the fatty acid synthetase to membrane bound enzymes.

We consider a spherical diffusion cell (radius $R$ ) containing randomly diffusing FAS molecules which are ideglly reflected by the surrounding membrane. Thus, macroscoplc concentration gradients do not exist and the average time $\tau^{\prime}(3)$ required by an FAS molecule to hit the membrane is governed by the self-diffusion of the FAS. (The index (3) is used for three-dimensional diffusion). $T_{(3)}^{\prime}$ is given by

$\tau_{(3)}^{\prime}=c R^{2} / D_{(3)}$

where $D_{(3)}$ denotes the coefficient of free (three-dimensional) diffusion $\left(D_{(3)}\left(\mathrm{H}_{2} \mathrm{O}, 25^{\circ}\right) \approx 2 \times 10^{-7} \mathrm{~cm}^{2} /\right.$ sec for the FAS). Lower and upper limits for $c$ are $1 / 20$ and 3/2. These values are obtained by averaging over the shortest and longest times of travel from all places in the cell to the enclosing membrane. In the following a mean value of $c=3 / 4$ will be used.

We are interested in the average time $\left(\tau^{\circ}\right)$ required for the trarisfer of palmitoyl CoA from the FAS to target molecules on the membrane for the following two cases: i) the transfor is accomplistied through direct collisions between the freely diffusing FAS molecules and the membrane bound targets $\left(\tau_{(3)}\right)$; ii) the palmitoyl CoA is incorporated randomly into the membrane (via collisions between the FAS and the membrane) and reaches the target molecules through lateral diffusion with in the plane of the membrane $\left(T_{(3 / 2)}\right)$. It will be assumed: (1) that the area of all targets is negligibly small compared to the rest of the inembrane, (2) that the targets represent "sinks" of infinite capacity and (3) that following the release of palmitoy! CoA from the FAS new palmitoyl COA molecules are synthesized in a time which is short compared to the transfer times $(\tau)$. The average time $\tau_{(3)}$ required by a freely diffusing FAS molecule to hit a target on the membrane is

$\tau_{(3)}=\tau_{(3)}^{\prime} / p_{t} f_{t}$

where $\rho_{\mathrm{r}}$ denotes the density of target molecules per $\mathrm{cm}^{2}$ of the membrane surface and $f_{t}$ is the surface area of one target molecule.

For the combined mechanism (free and lateral diffusion) the average lime of transfor is

$\tau_{(3 / 2)}=\tau_{(3)}^{\prime}+\tau_{(2)}$

where $T_{(2)}$ denotes the average time of lateral diffusion from an arbitrary place on the membrane to a target molecule. Since the target molecules represent sinks for the palmitoyl CoA, concentration gradients will be established within the plane of the membrane. For an exact treatment of the diffusion-problem within the membrane one has to start from Fick's second law of diffusion and assumptions must be made about the distribution and density of sinks and sources.

However, an approximate value for $\tau_{(2)}$ can be estimated easily if we consider the lateral diffusion of the palmitoyl $\mathrm{CoA}$ as a random walk problem (self-diffu: sion) involving a series of successive jumps of length $\lambda$ within the two-dimensional lattice of lipid molecules.

One derives (cf. [16])

$\tau_{(2)}=\frac{1}{2\left(r_{d}+r_{t}\right) / \rho_{t}}=\frac{\lambda}{4\left(r_{d}+r_{t}\right) \rho_{t} D_{(2)}}$

where / denutes the integrated diffusional path in 1 $\sec \left(l=D_{(2)} / 2 \lambda\right)$ and $\left(r_{d}+r_{t}\right)$ is the sum of the radii of the diffusing molecule (palmitoyl CoA) and the target molecule. Using the values $D_{(3)}=2 \times 10^{-7} \mathrm{~cm}^{2} / \mathrm{sec}$, $D_{(2)}=2 \times 10^{-8} \mathrm{~cm}^{2} / \mathrm{sec}, \lambda=8 \mathrm{~A}$ (cf. $[16]$ ) and assuming $\left(r_{d}+r_{t}\right) \approx 50 \mathrm{~A}$ we obtain for the iarii) $q$ of the transfer times:

$q=\frac{\dot{\tau}_{(3 / 2)}}{\tau_{(3)}}=f_{t} \rho_{t}\left(1+\frac{\lambda}{3 \rho_{t}\left(r_{d}+r_{t}\right)} \frac{1}{R^{2}} \frac{D_{(3)}}{D_{(2)}}\right) \approx J_{t}\left(\mu_{t}+\frac{1}{2 R^{2}}\right)$

For 3 given ratio of the diffusion coefficients $\left(D_{(3)} / D_{(2)} \propto 10\right.$ in our case) the ratio of the transfer times is determined by the area $\left(f_{t}\right)$ and density $\left(\rho_{t}\right)$ of the target molecules and by the radius, $R$, of the diffusion space. 
The combined transfer mechanism becomes mone favorable ( $q$ decreases) with decreasing density of targets and increasing size of the diffusion space. In . fig. 5 contour lines are drawn in an $R$ versis $p_{t}$ dia-: gram enclosing the regimes in which the combined ... mechanism is more favorable $(q<1)$. For a given area of the target molecules $\left(f_{t}\right)$ the combined mechanism is more favorable if $i)$ the radius of the diffusion 'space is larger than a critical value $R^{e r}$ and if ii) the density of target molecules is lower than a critical value $\rho_{t}^{e r}$.

Defining the "more favarable" regime by $q \leq 0.1$ we obtain $R^{e r} \approx 200 \AA, \rho_{t}^{r r}=10^{11} / \mathrm{cm}^{2}$ for $f_{t}=10^{4} \AA^{2}$; and $R^{c r}=80 \AA ; \rho_{t}^{c r}=10^{12} / \mathrm{cm}^{2}$ for : $f_{t}=10^{3} \mathrm{~A}^{2}$. This shows that in the case considered $\left(D_{(3)} / D_{(2)} \approx 10\right)$ an extremely wide range of parameters $\left(f_{t}, \rho_{t}, R\right)$ exists for which the combined mechanism (free and lateral diffusion) is more favourable than diffusion alone.

\section{References}

(1) W.C.:McMurray and W.L. Magee, Ann. Rev, Blochem. 41 (1972) 129.

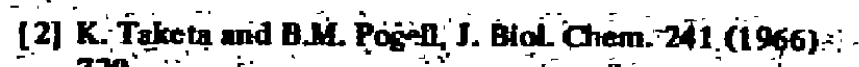
720. $\rightarrow$

[3] W.L Zahier, R.E Barden and W.W.Clelind Bjochin. Bfophys Act 164 (1968) 1, :

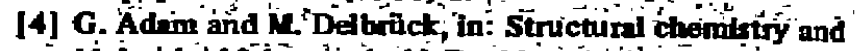

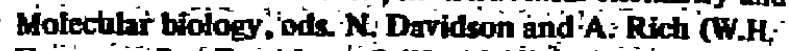
Frëemoin; Sar Francisco, Callif, 1968) p. 198.

[sj F. Lynen, in: Methods enzymol, ed, J.M.Loprengtein (Acad: Press, New York, 1969 ) 14, p. 17.

[6] EJ. Stmon and D. Shemin, J. Am. Chem. Soc. 75 (1953) 2520.

[7l H. Egigerer and F:Lyrẹi, Bochem-Z 335 (1962) 540

[8] T. Wieland, Naturwiss: 38 (1951) 384: : : : :-

[9I T. widud, Ampori. Chom. 65 (1953) (86.

[10] H.R. Kaback, in: Methods endym., ed W. Jakoby (Acad Press, Now York, 1971 ) 22, p. 99. .

111] G.'Lust ánd F. Lyaen, European 3. Bíochein. 7 (1968) 68

[12] G.K. Radda, Curr. Topkt Bioenorg 4 (1971) 81. -

[13] H. Trable; Naturwias 58.(1971) 277.-

[14] H. Trauble, in: Biomombrines 3 , eds. F. Kreuzer and J.F.G. Slegers (Plonum Prex, 1972) p. 127.

[15] B.D. Eadbrooke, R.M William and D. Chapmin, Biochem. Biophys Acit iso (1958) 333 .

[16] H. Tripbic and E. Selomann, J. Am. Chin. Soc 94 (1972) 4499.

[17] P. Devaux and H.M. AcConnell, J. Am. Chem. Soc. 94 (1972) 4475.

[18] W. Pirson, Doctoral Thesis, Munich 1970: 